\title{
Correction to: Treatment of Open-Angle Glaucoma and Ocular Hypertension with Preservative-Free Tafluprost/Timolol Fixed-Dose Combination Therapy: The VISIONARY Study
}

Francesco Oddone · Lucia Tanga · Péter Kóthy · Gábor Holló ·

The VISIONARY Study Group

Published online: June 21, 2020

(C) The Author(s) 2020

Correction to: Adv Ther (2020) 37:1436-1451

https://doi.org/10.1007/s12325-020-01239-8

In the original article, the The VISIONARY Study Group Principal Investigator Jose Garcia Medina (Hospital General Universitario Morales Meseguer, Murcia) should have appeared with his full name and surname (Jose Javier GarciaMedina). Thus, he was incorrectly identified in PubMed as Medina JG. The correct name should display as Garcia-Medina JJ.

\section{ACKNOWLEDGEMENTS}

VISIONARY Study Group Principal Investigators. Austria: Christoph Faschinger (LKH Universitaetsklinikum Graz); Sweden: Enping Chen (St Eriks Eye Clinic, Stockholm); Hungary: Gabor Holló (Semmelweis University,

The members of The VISIONARY Study Group are listed in Acknowledgements.

The original article can be found online at https://doi. org/10.1007/s12325-020-01239-8.

F. Oddone $(\bowtie) \cdot$ L. Tanga

IRCSS-Fondazione Bietti, Rome, Italy

e-mail: oddone-bietti@gmail.com

P. Kóthy · G. Holló

Department of Ophthalmology, Semmelweis

University, Budapest, Hungary
Budapest), Gabor Nemeth (Borsod-Abaúj-Zemplén Megyei Kórház Szemészeti, Miskolc), Gyorgy Bator (Markusovszky Egyetemi Oktató Kórház, Szombathely), Alexis Tsorbatzoglou (Szabolcs-Szatmár-Bereg County Hospital and University Teaching Hospital, Nyíregyhaza),Tamas Acs (Bács-Kiskun Megyei Kórház, Kecskemét), Maria Ferencz (Szent Imre Egyetemi Oktatókórház, Budapest), Zoltán Sohajda (Kenézy Gyula Kórház és Rendelőintéze, Debrecen), Jeno Toth (Fejér Megyei Szent György Egyetemi Oktató Kórház, Székesfehérvár), Veronika Volner (Uzsoki Utcai Kórház, Budapest), Gabor Vogt (Magyar Honvédség Egészségügyi Központ, Budapest), Zsolt Biro (PTE- Szemészeti Klinika, Pécs), Andrea Facskó (Szegedi Tudományegyetem Szent-Györgyi Albert Klinikai Központ, Szent-György), János Nemes (Megyei Flór Ferenc Kórház, Kistarcsa), Andras Berta (University of Debrecen, Debrecen), Ilona Elek (Bugát Pál Kórház, Gyöngyös); Ireland: Eugene Ng (The Whitfield Clinic Butlerstown, Waterford); Italy: Francesco Oddone (IRCSS-Fondazione Bietti, Rome), Gemma Rossi (IRCCS-Fondazione Policlinico San Matteo, Pavia), Luca Rossetti (Ospedaliera San Paolo, Milan), Michele Vetrugno (Università di Bari, Bari), Michele Iester (Università Degli Studi Di Genova, Genoa), Giorgio Marchini (Ospedale Civile Maggiore Borgo Trento, Verona), Vincenzo Scorcia (Università degli studi Magna Græcia, Catanzaro), Giovanni Staurenghi (Ospedale Luigi Sacco, Milan), Carlo Cagini 
(Università degli Studi di Perugia, Perugia), Tommaso Salgarello (Fondazione Policlinico Gemelli, Rome), Paolo Bettin (IRCCS Ospedale San Raffaele, Milan), Michele Figus (Ospedale Cisanello, Pisa), Gian Luca Scuderi (Ospedale Sant'Andrea, Rome), Stefano De Cilla (Azienda Ospedaliera Universitaria Maggiore della Carita Presidio Ospedaliero San Rocco, Novara); Latvia: Iveta Grundmane (Grund-opt Ltd, Valmiera), Nora Linavska (LENS Ltd, Liepaja), Lasma Volksone (Lavolks Ltd, Riga), Guna Laganovska (P. Stradiņš Clinical University Hospital, Riga), Kristine Baumane (Riga East Clinical University Hospital, Riga); Netherlands: Hans Lemij (Rotterdam Ophthalmic Institute, Rotterdam); Norway: Kjell Gunnar Gundersen (Dr Kjell Gunnar Gundersen MD, Haugesund); Russia: Marina Zimina (LLC Vzglyad, Leningradskaya), Valery Erichev (Federal State Budgetary Scientific Institution Scientific, Research Institute of Eye Diseases, Moscow), Elmira Adbulaeva (State Autonomous Institution of Healthcare Republican Clinical Ophthalmological Hospital of the Ministry of Healthcare of the Republic of Tatarstan, Republic of Tatarstan), Elena Karlova (State Budgetary Institution of Healthcare Samara Regional Clinical Ophthalmological Hospital, Samara), Ekaterina Zakharova (RKOB Sverdlova, Yakutsk), Irina Panova (S. Fyodorov Eye Microsurgery Federal State Institution, Saint Petersburg), Boris Malyugin (Sv. Fyodorov's Eye Microsurgery Complex, Moscow); Spain: Inaki Rodriguez Aguirrec (H.U. Donostia, Guipuzcoa), Fernando Lopez-Lopez (Instituto Oftalmologico Gomez-Ulla, Galicia), Antonio Moreno Valladares (Hospital Ntra. Sra. del Perpetuo Socorro, Albacete), Javier Benitez del Castillo (Hospital Universitario de Jerez, Cadiz), Rafael Gimenez (Hospital Reina Sofia, Cordoba), Maria Parrilla Vallejo (Hospital La Macarena, Seville), Jose Javier Garcia-Medina (Hospital General Universitario Morales Meseguer, Murcia), Alfonso Anton Lopez (Institut Català de Retina, Barcelona), Sergio Torregrosa (Hospital Punta de
Europa, Cadiz), Jorge Loscos (Hospital German Trias i Pujol, Barcelona); Denmark: Miriam Kolko (Rigshospitalet Valdemar Hansensvej, Glostrup); UK: Ejaz Ansari (Maidstone Hospital Hermitage, Maidstone, Kent), David Broadway (Norfolk and Norwich University Hospital, Norwich, Norfolk), Katharine Claridge (Royal Cornwall Hospital, Truro, Cornwall), Simon Ruben (Southend University Hospital NHS Foundation Trust, Westcliff-On-Sea, Essex), James Kirwan (Queen Alexandra Hospital, Portsmouth Hospitals NHS Trust, Portsmouth, Hampshire), Anca Nita (Royal Victoria Infirmary, The Newcastle Upon Tyne Hospitals NHS Foundation Trust, Newcastle Upon Tyne), Micheal Smith (Royal Devon \& Exeter NHS Foundation Trust, Exeter, Devon), Areeb Moosavi (Milton Keynes University Hospital NHS Foundation Trust, Milton Keynes), Anthony J W King (Nottingham University Hospitals NHS Trust, Nottingham), Matthew Kinsella (Buckinghamshire Healthcare NHS Trust, Stoke Mandeville Hospital, Buckinghamshire).

Open Access. This article is licensed under a Creative Commons Attribution-NonCommercial 4.0 International License, which permits any non-commercial use, sharing, adaptation, distribution and reproduction in any medium or format, as long as you give appropriate credit to the original author(s) and the source, provide a link to the Creative Commons licence, and indicate if changes were made. The images or other third party material in this article are included in the article's Creative Commons licence, unless indicated otherwise in a credit line to the material. If material is not included in the article's Creative Commons licence and your intended use is not permitted by statutory regulation or exceeds the permitted use, you will need to obtain permission directly from the copyright holder. To view a copy of this licence, visit http://creativecommons.org/licenses/by$\mathrm{nc} / 4.0 /$. 